\title{
SEMIPRIME SEMIGROUP RINGS AND A PROBLEM OF
}

\section{J. WEISSGLASS}

\author{
by MARK L. TEPLY
}

(Received 19 March, 1979)

If $R$ is a ring and $S$ is a semigroup, the corresponding semigroup ring is denoted by $R[S]$. A ring is semiprime if it has no nonzero nilpotent ideals. A semigroup $S$ is a semilattice $P$ of semigroups $S_{\alpha}$ if there exists a homomorphism $\varphi$ of $S$ onto the semilattice $P$ such that $S_{\alpha}=\alpha \varphi^{-1}$ for each $\alpha \in P$.

In [4] J. Weissglass proves the following result.

THeOREM. Suppose that $R$ is a commutative ring with identity element and that $S$ is a commutative semigroup such that a power of each element lies in a subgroup. Then $R[S]$ is semiprime if and only if $S$ is a semilattice $P$ of groups $S_{\alpha}$, and $R\left[S_{\alpha}\right]$ is semiprime for each $\alpha \in P$.

Then Weissglass asks [4, Question 9, page 477] if the commutativity of $R$ can be removed from the hypothesis of his theorem. The purpose of this note is to answer his question affirmatively.

Given a ring $R$ and a semigroup $S$, the support of $x=\sum_{s \in S} r_{s} s \in R[S]$, denoted by $\operatorname{supp} x$, is defined to be the set $\left\{s \in S \mid r_{s} \neq 0\right\}$. For a set $X,|X|$ denotes the cardinality of $X$.

LEMMA 1. Let $R$ be a ring with identity element, and let $S$ be a commutative semigroup. Assume that the group $G$ is an ideal of $S$ and that every element of $S$ has a power in $G$. Let $A$ be a nonzero ideal of $R[S]$ such that $A \cap R[G]=0$. Then there exists a nonzero element $y=\sum_{i=1}^{n} r_{i} s_{i} \in A\left(r_{i} \in R, s_{i} \in S\right)$ such that $y r s_{j}=0$ for each $r \in R$ and each $j \leq n$.

Proof. Let $m=\min \{j \mid 0 \neq x \in A$ and $|(\operatorname{supp} x) \cap(S-G)|=j\}$. Since $A \cap R[G]=0$, then $m \geq 1$. Let $y=\sum_{i=1}^{n} r_{i} s_{i} \in A-\{0\}$ be chosen such that

$$
\left\{s_{1}, s_{2}, \ldots, s_{m}\right\}=(\operatorname{supp} y) \cap(S-G)
$$

and

$$
\left\{s_{m+1}, \ldots, s_{n}\right\} \subseteq G \text { if } m<n .
$$

Let $k$ be a positive integer such that $k \leq m$, and consider the condition $y s_{j}=0$ for $j<k$. This condition is vacuously satisfied when $k=1$; so assume that the condition holds for some $k \geq 1$. Since a power, say $s_{k}^{t}$, of $s_{k}$ is in the idéal $G$ of $S$, then $y s_{k}^{t} \in R[G]$. But $y s_{k}^{t} \in A$, since $y \in A$. Hence $y s_{k}^{t}=0$. Thus there is a least nonnegative integer $h$ such that $y s_{k}^{h+1}=0$. (If $h=0$, let $s_{k}^{0}=1 \in R$ for notational convenience.) Then by the choice of $m$ and $h$, we have that $s_{1} s_{k}^{h}, s_{2} s_{k}^{h}, \ldots, s_{m} s_{k}^{h}$ are distinct elements of $S-G$, and $s_{i} s_{k}^{h} \in G$ for

Glasgow Math. J. 21 (1980) 131-134. 
$i>m$. Since $S$ is commutative,

$$
\left(\sum_{i=1}^{n} r_{i} s_{i} s_{k}^{h}\right) s_{i} s_{k}^{h}=\left(\sum_{i=1}^{n} r_{i} s_{i}\right) s_{j} s_{k}^{2 h}=y s_{j} s_{k}^{2 h}=0
$$

for $j \leq k$. Thus, if we replace $s_{i}$ by $s_{i} s_{k}^{h}$ in our original expression for $y$, we may assume that $y=\sum_{i=1}^{n} r_{i} s_{i} \in A-\{0\}, s_{i} \in S-G$ for $i \leq m, s_{i} \in G$ for $i>m$, and $y s_{j}=0$ for $j \leq k$. By induction, we may assume that $y s_{j}=0$ for $j \leq m$. Since $G$ is an ideal of $S$, we also have $y s_{j} \in A \cap R[G]=0$ for each $j>m$.

Let $j \in\{1, \ldots, n\}$. Write $T=\left\{s_{i} s_{j} \mid i=1, \ldots, n\right\}$ and, for each $t \in T$, let $I_{t}=\left\{i \mid s_{i} s_{j}=t\right\}$.

Since $y s_{j}=0$, we have that, for all $t \in T, \sum_{i \in I_{i}} r_{i}=0$. Hence, for all $r \in R$,

$$
0=\sum_{t \in T}\left(\sum_{i \in I_{t}} r_{i}\right) r t=\sum_{i=1}^{n} r_{i} r\left(s_{i} s_{j}\right)=\left(\sum_{i=1}^{n} r_{i} s_{i}\right) r s_{j}=y r s_{j} .
$$

Let $P$ be a semilattice whose natural order is indicated by $\leq$, and let $S$ be a semilattice $P$ of semigroups $S_{\alpha}$. Then there exist ideal extensions $D_{\alpha}$ of $S_{\alpha}(\alpha \in P)$ and homomorphisms $\varphi_{\alpha, \beta}: S_{\alpha} \rightarrow D_{\beta}(\beta \leq \alpha)$ satisfying the following conditions:

(a) $\varphi_{\alpha, \alpha}$ is the identity map on $S_{\alpha}$;

(b) $\left(S_{\alpha} \varphi_{\alpha, \alpha \beta}\right)\left(S_{\beta} \varphi_{\beta, \alpha \beta}\right) \subseteq S_{\alpha \beta}$;

(c) if $\alpha \beta>\gamma$, then for all $a \in S_{\alpha}$ and $b \in S_{\beta},\left[\left(a \varphi_{\alpha, \alpha \beta}\right)\left(b \varphi_{\beta, \alpha \beta}\right)\right] \varphi_{\alpha \beta, \gamma}=\left(a \varphi_{\alpha, \gamma}\right)\left(b \varphi_{\beta, \gamma}\right)$;

(d) $S$ is the disjoint union of the $S_{\alpha}(\alpha \in P)$;

(e) if $a \in S_{\alpha}$ and $b \in S_{\beta}$, then multiplication in $S$ is determined by

$$
a b=\left(a \varphi_{\alpha, \alpha \beta}\right)\left(b \varphi_{\beta, \alpha \beta}\right) \in S_{\alpha \beta} .
$$

For more details, see Section III.7 of [2]. We note that each $\varphi_{\alpha, \beta}$ has a natural extension to a ring homomorphism from $R\left[S_{\alpha}\right]$ to $R\left[D_{\beta}\right]$ :

$$
\sum_{s \in S_{\alpha}} r_{s} s \rightarrow \sum_{s \in S_{\alpha}} r_{s}\left(s \varphi_{\alpha, \beta}\right)
$$

We also denote this extension by $\varphi_{\alpha, \beta}$ for convenience.

Lemma 2. Let $R$ be a ring with identity element, and let $S$ be a semilattice $P$ of commutative semigroups $S_{\alpha}$. Let $\sigma \in P$ and $y=\sum_{i=1}^{n} r_{i} s_{i} \in R\left[S_{\sigma}\right]$ be such that $y s_{j}=0$ for each $r \in R$ and each $j \leq n$. Then the principal ideal $B$ of $R[S]$ generated by y satisfies $B^{2}=0$.

Proof. Every element of $B^{2}$ is a sum of terms, each of which contains at least one of the following factors: $y^{2}$ or $y . r s . y$, where $r \in R$ and $s \in S_{\alpha}$ for some $\alpha \in P$.

But $y^{2}=\sum_{i=1}^{n} y r_{i} s_{i}=0$ by our choice of $y$. Moreover, if $r \in R$ and $s \in S_{\alpha}$ then, since $S_{\alpha \sigma}$ 
is commutative and $\varphi_{\sigma, \alpha \sigma}$ is a homomorphism, we have

$$
\begin{aligned}
y . r s . y & =\left(\sum_{i=1}^{n} r_{i} s_{i}\right) . r s \cdot\left(\sum_{i=1}^{n} r_{i} s_{i}\right) \\
& =\sum_{i, j} r_{i} r_{j}\left(s_{i} \varphi_{\sigma, \alpha \sigma}\right)\left(s \varphi_{\alpha, \alpha \sigma}\right)\left(s_{j} \varphi_{\sigma, \alpha \sigma}\right) \\
& =\sum_{i, j} r_{i} r_{j}\left(\left(s_{i} s_{j}\right) \varphi_{\sigma, \alpha \sigma}\right)\left(s \varphi_{\alpha, \alpha \sigma}\right) \\
& =\left[\sum_{j=1}^{n}\left(\left(\sum_{i=1}^{n} r_{i} s_{i}\right) r r_{j} s_{j}\right) \varphi_{\sigma, \alpha \sigma}\right]\left(s \varphi_{\alpha, \alpha \sigma}\right) \\
& \left.=\left[\sum_{j=1}^{n}\left(y\left(r r_{j}\right) s_{j}\right) \varphi_{\sigma, \alpha \sigma}\right)\right]\left(s \varphi_{\alpha, \alpha \sigma}\right)=0
\end{aligned}
$$

by our choice of $y$.

It follows that $B^{2}=0$ as desired.

Lemma 3. Let $R$ be a ring with identity element, and let $S$ be a semilattice $P$ of commutative semigroups $S_{\alpha}$. Let $\sigma \in P$, and assume that the group $G$ is an ideal of $S_{\sigma}$. Let $A$ be a nilpotent ideal of $R\left[S_{\sigma}\right]$ such that $A^{2}=0$. Then the principal ideal $C$ of $R[S]$ generated by any element of $A \cap R[G]$ satisfies $C^{2}=0$.

Proof. Let $x=\sum_{i=1}^{n} r_{i} s_{i} \in A \cap R[G]$ with $\left\{s_{1}, s_{2}, \ldots, s_{n}\right\} \subseteq G$, and let $x$ generate $C$. Since $x^{2}=0$, then every element of $C^{2}$ is a sum of terms, each of which contains a factor of the form $x, r s . x$, where $r \in R$ and $s \in S_{\alpha}$ for some $\alpha \in P$. Let $e$ be the identity element of $G$, let $r \in R$, and let $s \in S_{\alpha}$ for some $\alpha \in P$. Since $S_{\alpha \sigma}$ is commutative and $\varphi_{\sigma, \alpha \sigma}$ is a homomorphism, we have

$$
\begin{aligned}
x . r s . x & =\left(\sum_{i=1}^{n} r_{i} s_{i}\right) \cdot r s \cdot\left(\sum_{i=1}^{n} r_{i} s_{i}\right) \\
& =\sum_{i, j} r_{i} r_{j}\left(s_{i} \varphi_{\sigma, \alpha \sigma}\right)\left(s \varphi_{\alpha, \alpha \sigma}\right)\left(s_{j} \varphi_{\sigma, \alpha \sigma}\right) \\
& =\left[\left(\sum_{i, j} r_{i} r r_{j} s_{i} s_{j}\right) \varphi_{\sigma, \alpha \sigma}\right]\left(s \varphi_{\alpha, \alpha \sigma}\right) \\
& =\left[\left(\sum_{i=1}^{n} r_{i} s_{i}\right)(r e)\left(\sum_{j=1}^{n} r_{j} s_{i}\right)\right] \varphi_{\sigma, \alpha \sigma} \cdot\left(s \varphi_{\alpha, \alpha \sigma}\right) \\
& \subseteq\left(A^{2}\right) \varphi_{\sigma, \alpha \sigma} \cdot\left(s \varphi_{\alpha, \alpha \sigma}\right) \\
& =0 .
\end{aligned}
$$

It follows that $C^{2}=0$ as desired.

We are now ready for our main result. 
THEOREM. Let $R$ be a ring with identity element, and let $S$ be a commutative semigroup such that a power of each element of $S$ lies in a subgroup. Then $R[S]$ is semiprime if and only if $S$ is a semilattice $P$ of groups $S_{\alpha}$, and $R\left[S_{\alpha}\right]$ is semiprime for each $\alpha \in P$.

Proof. By [1, §4.3, Exercise 5] the hypothesis on $S$ forces $S$ to be a semilattice $P$ of semigroups $S_{\alpha}$, where each $S_{\alpha}$ contains a group ideal $G_{\alpha}$ such that $S_{\alpha} / G_{\alpha}$ is a nil semigroup.

Let $R[S]$ be semiprime. Suppose that there exists $\sigma \in P$ such that $R\left[S_{\sigma}\right]$ is not semiprime. Then $R\left[S_{\sigma}\right]$ contains a nonzero nilpotent ideal $A$ such that $A^{2}=0$. If $A \cap R\left[G_{\sigma}\right]=0$, then $R[S]$ has a nonzero nilpotent ideal $B$ by Lemmas 1 and 2 ; if $A \cap R\left[G_{\sigma}\right] \neq 0$, then $R[S]$ has a nonzero nilpotent ideal $C$ by Lemma 3. Consequently, each $R\left[S_{\sigma}\right]$ must be semiprime to avoid a contradiction. It now follows from [4, Lemma 4] that each $S_{\alpha}$ is a group.

The converse follows from [3, Theorem 1] or [4, Corollary 1].

\section{REFERENCES}

1. A. H. Clifford and G. B. Preston, The algebraic theory of semigroups, Vol. I, Math. Surveys No. 7, Amer. Math. Soc. (Providence, R. I, 1961).

2. M. Petrich, Introduction to semigroups (Merrill, Columbus, 1973).

3. M. L. Teply, E. G. Turman, and A. Quesada, On semisimple semigroup rings, Proc. Amer. Math. Soc. (to appear).

4. J. Weissglass, Semigroup rings and semilattice sums of rings, Proc. Amer. Math. Soc. 39 (1973), 471-478.

UNIVERSITY OF FLORIDA

Gainesville, Florida 32611 